\title{
The effect of probiotic, prebiotic, and polyphenol interventions on cardiovascular disease risk markers: findings from the CABALA study
}

\author{
S.M. Alzoufairi ${ }^{1}$, R.A.G. Pushpass ${ }^{1}$, C. Pedersen ${ }^{1}$, K.G. Jackson ${ }^{1}$ and J.A. Lovegrove ${ }^{1}$ \\ ${ }^{1}$ Hugh Sinclair Unit of Human Nutrition, Department of Food and Nutritional Sciences, Harry Nursten Building, \\ Whiteknights, University of Reading, Reading, UK
}

Cardiovascular diseases (CVD) are a major cause of mortality and disability in the $\mathrm{UK}^{(1)}$. Since diet and low density lipoprotein cholesterol (LDL-C) are both important modifiable CVD risk markers, there is considerable interest in dietary strategies which can reduce LDL-C levels for disease prevention ${ }^{(2)}$. Changes in gut microbiota composition during supplementation with dietary fibres, polyphenols and probiotics have been proposed to impact on fasting total serum cholesterol levels via effects on bile acid metabolism. However, further studies are needed to confirm the effects of these interventions on CVD risk markers and whether they are related to changes in circulating bile acids.

The aim of the Circulating Bile Acids as Biomarkers of Metabolic Health- Linking microbiota, Diet and Health (CABALA) study was to investigate how chronic consumption of probiotics (lactobacillus reuteri), prebiotics (oats) and apples (rich in polyphenols/ fibre) impact on CVD risk markers compared with a control intervention. In this single-blind, chronic parallel trial, 64 adults aged between 25-70 years and BMI 20-32 kg/m2 were randomised based on age, sex, BMI and serum total cholesterol (TC) to one of four groups: i) $40 \mathrm{~g} /$ day of porridge oats with two placebo capsules, ii) two Renetta Canada apples/day with two placebo capsules, iii) $40 \mathrm{~g} /$ day of cornflakes with two probiotic capsules or iv) $40 \mathrm{~g} /$ day of cornflakes with two placebo capsules (control) for 8 weeks. Fasting blood samples were collected at the beginning and end of the intervention period for the measurement of the lipid profile including TC, high-density lipoprotein-cholesterol (HDL-C), triacylglycerol and apolipoprotein (apo)B. LDL-C was estimated using the Friedewald formula.

Sixty-one volunteers completed the study (mean $\pm \mathrm{SD}$, age $25 \pm 12$ y and BMI $24.8 \pm 3.3 \mathrm{~kg} / \mathrm{m} 2$ ). At week 0 (baseline), fasting lipid concentrations and TC:HDL-C ratio were similar between the intervention groups. Relative to baseline, there was a significant reduction in fasting apoB after consuming apples for 8 weeks compared with the control intervention $(P=0.034)$. The other fasting lipids and TC:HDL-C ratio were not found to be different over the 8 week period between the apples $(n=16)$, oats $(n=15)$, probiotic $(n=$ $15)$ or control $(n=15)$ interventions.

Whilst our previous study reported beneficial effects of consuming two Renetta Canada apples/day on fasting TC, LDL-C and triacylglycerol in mildly hypercholesterolaemic adults(3), the current study found only a significant apoB lowering effect after the apples, with little impact evident on the other fasting lipids. Differences between study finding may have been due to the population group studied or the comparison with other dietary components, which are also known to impact on circulating blood lipids and the gut microbiome.

\section{Acknowledgements}

The CABALA study was supported by BBSRC (BB/P028209/1), with food in-kind from Pepsico and UAS Lab's The Probiotic Company.

\section{References}

1. Bhatnagar P, Wickramasinghe K, Williams J, et al. (2015) Heart 101, 1182-1189.

2. Lewington S, Whitlock G, Clarke R, et al. (2008) Lancet 370 (9602), 1829-1839.

3. Koutsos A, Riccadonna S, Ulaszewska MM et al. (2020) Am J Clin Nutr 1;111(2), 307-318. 